NASA Technical Memorandum 105904

$1 N-64$

ICOMP-93-06

\title{
Large-Scale Computation of Incompressible Viscous Flow by Least-Squares \\ Finite Element Method
}

$=\ldots$ Bo-nan Jiang

Institute for Computational Mechanics in Propulsion

Lewis Research Center

Cleveland, Ohio

\section{T.L. Lin}

Livermore Software Technology Corporation

Livermore, California

and

Louis A. Povinelli

Lewis Research Center

Cleveland, Ohio
F

(NASA-TM-105904) LARGE-SCALE COMPUTATION OF INCOMPRESSIBLE VISCOUS FLOW BY LEAST-SQUARES FINITE ELEMENT METHOD (NASA)
N93-22825

$22 p$

\section{G3/64 0151397}

March 1993

1
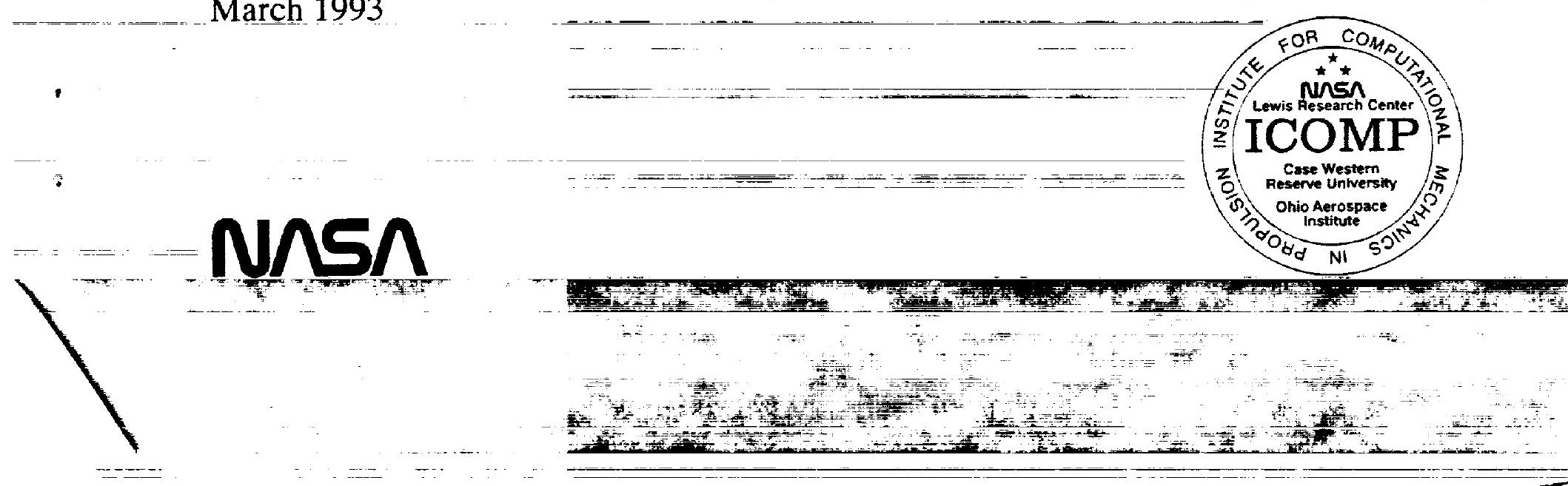


\title{
LARGE-SCALE COMPUTATION OF INCOMPRESSIBLE VISCOUS FLOW BY LEAST-SQUARES FINITE ELEMENT METHOD
}

\author{
Bo-nan Jiang \\ ICOMP, MS 5-3, NASA Lewis Research Center \\ Cleveland, Ohio 44135, USA \\ T.L. Lin \\ Livermore Software Technology Corporation \\ Livermore, California 94550, USA \\ Louis A. Povinelli \\ Internal Fluid Mechanics Division, NASA Lewis Research Center \\ Cleveland, Ohio 44135, USA
}

\begin{abstract}
The least-squares finite element method(LSFEM) based on the velocity-pressurevorticity formulation is applied to large-scale/three-dimensional steady incompressible Navier-Stokes problems. This method can accommodate equal-order interpolations, and results in symmetric, positive definite algebraic system which can be solved effectively by simple iterative methods. The first-order velocity-Bernoulli function-vorticity formulation for incompressible viscous flows is also tested. For three-dimensional cases, an additional compatibility equation, i.e., the divergence of vorticity vector should be zero, is included to make the first-order system elliptic. The simple substitution or the Newton's method is employed to linearize the partial differential equations, the LSFEM is used to obtain discretized equations, and the system of algebraic equations is solved using the Jacobi preconditioned conjugate gradient method which avoids formation of either element or global matrices (matrix-free) to achieve high efficiency. To show the validity of this method for large-scale computation, we give numerical results for $2 \mathrm{D}$ driven cavity problem at $\mathrm{Re}=$ 10000 with $408 \times 400$ bilinear elements. The flow in a $3 \mathrm{D}$ cavity is calculated at $\operatorname{Re}=100$, 400 , and 1,000 with $50 \times 50 \times 50$ trilinear elements. The Taylor-Görtler-like vortices are observed for $\operatorname{Re}=1,000$.
\end{abstract}




\section{Introduction}

Although significant progress has been made in the finite element solution of incompressible viscous flow problems, development of more efficient methods is still needed before large-scale computation of 3D problems becomes feasible. This paper presents such a development.

The most popular finite element method for the solution of incompressible NavierStokes equations is the classic Galerkin mixed method based on the velocity-pressure formulation, e.g., see Refs. [1-10]. One of notorious difficulties in the mixed method is the satisfaction of the Ladyzhenskaya-Babuska-Brezzi (LBB) condition which requires the use of different elements to interpolate the velocity and the pressure in order to obtain a stable scheme. For two-dimensional problems quite a few convergent pairs of velocity and pressure elements have been developed, however most of these combinations employ basis functions that are not convenient to implement. For three-dimensional problems, this difficulty becomes more severe and only rather elaborate constructions can pass the LBB test. Another difficulty is due to the lack of symmetry and positive definiteness of the linear equations arising from the classic mixed method. Iterative methods for solving this kind of linear system have been hard to come by. As Gresho, Lee and Sani[11] pointed out "there is currently no iterative solution method which can be guaranteed to work on the discretized, primitive-variable incompressible NS equations". Therefore, direct Gaussian elimination has been considered the only viable method for solving these systems. Unfortunately, for three-dimensional problems the computer resources required by a direct method are beyond the capacity of present hardware.

One way to circumvent the LBB condition is to modify the classical Galerkin mixed functional by appending some least-squares terms as Hughes and co-workers[12] did for their mixed Galerkin/least squares method. However, this type of method requires the choice of parameters, which depend on the mesh size of individual elements, and results in nonsymmetric matrices, which are still hard to deal with. An alternative approach to the use of equal-order elements (or unstaggered grids in a finite difference context) is to add the Laplacian of the pressure term and the divergence of the momentum into the continuity equation and to add a vector identity which relates the velocity and the vorticity into the momentum equations. Then standard finite volume, finite difference and Galerkin finite element methods can be employed to discretize the equations. This approach is proposed by Hafez and Soliman[13], and is essentially related to the mixed Galerkin/least-squares method.

In recent years a great deal of attention has been drawn to projection methods which have numerous aliases: splitting methods, fractional step methods, pressure correction methods, velocity correction methods and generalized and simplified marker-andcell(GSMAC) methods. This family of methods was originated with the work of Chorin[14] in a finite difference setting, and was transferred to the finite element version, e.g., see 
the studies by Gresho[15], Ramaswamy[16], Shimura and Kawahara[17], and Yagawa and Eguchi[18]. In these methods, the pressure computation is uncoupled with that of the velocity. The velocity is updated by explicit, implicit, or semi-implicit time-marching, and the pressure is obtained by solving the Poisson equation. These methods generally require less execution time and storage than the classical mixed Galerkin methods. This type of method should be classified as the mixed Galerkin method, and the LBB condition still should be respected.

In the category of projection methods we should especially mention adaptive $h p$ methods developed by Oden and co-workers[19]. Their techniques can vary simultaneously the mesh size $h$ and the spectral order $p$ of elements to produce high resolution of flow features with a relatively small amount of computation. Their techniques combine some new general multistep schemes based on high-order characteristic methods with pressure projections which are handled through a combination of exterior penalty methods and an adaptive pressure correction technique.

For the solution of incompressible Navier-Stokes equations, we have been developing a least-squares finite element method (LSFEM) [20-22]. This method is based on the firstorder velocity-pressure-vorticity formulation. Using $C^{0}$ finite elements to discretize the equations and minimizing the $L_{2}$ norm of the equation residuals lead to a symmetric and positive-definite algebraic system which can be effectively solved by simple matrix-free iterative methods. This is a minimization problem rather than a saddle point problem, and the choice of elements is thus not subject to the restriction of the LBB condition. In other words, all variables can be interpolated by the same element. This method is free of any parameters. There is neither added dissipation or upwinding, that is, the LSFEM is clean and robust. Since no derivatives are involved in boundary conditions, and only physical boundary conditions are imposed, the implementation of boundary conditions is extremely easy. In this method, all unknown variables (velocity, pressure and vorticity) are solved simultaneously, and there is no complicated iteration between them.

The capabilities of the LSFEM have been further shown by Lefebvre et al.[23] for two-dimensional unstructured triangular meshes, and by Tang and Tsang [24] for twodimensional time-dependent flows with thermal convection.

In the present paper the LSFEM is extended to large scale/three-dimensional computation. Following this introduction, the velocity-pressure-vorticity formulation for incompressible Navier-Stokes problems is presented in Section 2. An alternative velocityBernoulli function-vorticity formulation is discussed in Section 3. The numerical procedures for solving the first-order equations are addressed in Section 4. The numerical results for $2 \mathrm{D}$ and $3 \mathrm{D}$ cavity flows are contained in Section 5 . Concluding remarks are given in 
Section 6.

\section{Velocity-Pressure-Vorticity Formulation}

Let us consider the following steady-state incompressible Navier-Stokes problem in a bounded $2 \mathrm{D}$ or $3 \mathrm{D}$ domain $\Omega$ : Find the velocity $\bar{u}=(u, v, w)$ and the pressure $p$ such that

$$
\begin{array}{ll}
\nabla \cdot \bar{u}=0 & \text { in } \Omega, \\
\bar{u} \cdot \nabla \bar{u}+\nabla p-\frac{1}{R e} \Delta \bar{u}=\bar{f} & \text { in } \Omega .
\end{array}
$$

Here all variables are nondimensionalized, $\bar{f}=\left(f_{x}, f_{y}, f_{z}\right)$ is the body force, and $R e$ denotes the Reynolds number, defined as

$$
R e=\frac{U L}{\nu},
$$

where $L$ is a reference length, $U$ a reference velocity and $\nu$ the kinematic viscosity. Of course, boundary conditions should be specified to complete the definition of the boundary value problem.

Since the momentum equations( $1 \mathrm{~b}$ ) involve the second-order derivatives of velocity, direct application of the least-squares method requires the use of inconvenient $C^{1}$ elements and produces matrices with large condition number. In order to use the LSFEM with $C^{0}$ elements, we have to consider the governing equations of incompressible flow in the form of a first-order system. Therefore, we introduce the vorticity $\bar{\omega}=\left(\omega_{x}, \omega_{y}, \omega_{z}\right)=\nabla \times \bar{u}$ as an independent unknown vector, and rewrite the incompressible Navier-Stokes equations in the following first-order quasi-linear velocity-pressure-vorticity formulation:

$$
\begin{gathered}
\nabla \cdot \bar{u}=0, \\
\bar{u} \cdot \nabla \bar{u}+\nabla p+\frac{1}{R e} \nabla \times \bar{\omega}=\bar{f}, \\
\bar{\omega}-\nabla \times \bar{u}=0 .
\end{gathered}
$$

The first-order system (2a),(2b) and (2c) has an odd number (seven) of unknowns, i.e. $\left(u, v, w, p, \omega_{x}, \omega_{y}, \omega_{z}\right)$, and an odd number (seven) of equations, i.e. one continuity equation, three momentum equations, and three definitions of the vorticity components. When we classify this first-order system(assuming that the convective terms have already been linearized), we calculate the eigenvalues of a corresponding $7 \times 7$ coefficient matrix. Obviously, this matrix has at least one real eigenvalue which means that this first-order system cannot be elliptic in the ordinary sense. Therefore, the least-squares finite element 
method based on (2a),(2b) and (2c) cannot have an optimal rate of convergence for all unknown variables. In order to avoid this trouble, we add the following compatibility condition into the system:

$$
\nabla \cdot \bar{\omega}=0,
$$

so that we have seven unknowns and eight equations, and the ellipticity can be proved by using the same technique as discussed in [25].

For Cartesian co-ordinates, the system (2) is given as

$$
\begin{gathered}
\frac{\partial u}{\partial x}+\frac{\partial v}{\partial y}+\frac{\partial w}{\partial z}=0 \\
u \frac{\partial u}{\partial x}+v \frac{\partial u}{\partial y}+w \frac{\partial u}{\partial z}+\frac{\partial p}{\partial x}+\frac{1}{R e}\left(\frac{\partial \omega_{z}}{\partial y}-\frac{\partial \omega_{y}}{\partial z}\right)=f_{x} \\
u \frac{\partial v}{\partial x}+v \frac{\partial v}{\partial y}+w \frac{\partial v}{\partial z}+\frac{\partial p}{\partial y}+\frac{1}{R e}\left(\frac{\partial \omega_{x}}{\partial z}-\frac{\partial \omega_{z}}{\partial x}\right)=f_{y} \\
u \frac{\partial w}{\partial x}+v \frac{\partial w}{\partial y}+w \frac{\partial w}{\partial z}+\frac{\partial p}{\partial z}+\frac{1}{R e}\left(\frac{\partial \omega_{y}}{\partial x}-\frac{\partial \omega_{x}}{\partial y}\right)=f_{z} \\
\omega_{x}+\frac{\partial v}{\partial z}-\frac{\partial w}{\partial y}=0 \\
\omega_{y}+\frac{\partial w}{\partial x}-\frac{\partial u}{\partial z}=0 \\
\omega_{z}+\frac{\partial u}{\partial y}-\frac{\partial v}{\partial x}=0 \\
\frac{\partial \omega_{x}}{\partial x}+\frac{\partial \omega_{y}}{\partial y}+\frac{\partial \omega_{z}}{\partial z}=0
\end{gathered}
$$

Since the system is of first-order, the boundary conditions are thus very simple, and do not involve the derivatives of unknowns. Let $\left(\Gamma_{1}, \Gamma_{2}, \Gamma_{3}, \Gamma_{4}, \Gamma_{5}\right)$ denote the pieces of the boundary surface $\Gamma$. The unit outward normal vector to $\Gamma$ is denoted by $\bar{n}$, and the tangential vectors to $\Gamma$ by $\bar{\tau}_{1}$ and $\bar{\tau}_{2}$. We may consider, for instance, the following boundary conditions:

(a) $u=0, v=0, w=0$ on $\Gamma_{1}$ (the wall);

(b) $u=$ constant, $v=0, w=0$ on $\Gamma_{2}$ (the far field);

(c) $u=$ given, $v=0, w=0$ on $\Gamma_{3}$ (the well developed inflow or outflow);

(d) $u_{\tau 1}=0, u_{\tau 2}=0, p=$ constant on $\Gamma_{5}$ (the outflow);

(e) $u_{n}=0, \omega_{\tau 1}=0, \omega_{\tau 2}=0, p=$ constant on $\Gamma_{4}$ (the free surface). 
We note that in most cases the specification of boundary conditions for the vorticity components is not necessary. At solid wall and well developed inflow or outflow boundaries, we prescribe only the velocity components, no vorticity is involved.

\section{Velocity-Bernoulli Function-Vorticity Formulation}

The system (2) is not the only way to write a first-order formulation by using the vorticity as an independent unknown vector[26]. By introducing the Bernoulli function(the total enegy) $b=p+\frac{1}{2}\left(u_{1}^{2}+u_{2}^{2}+u_{3}^{2}\right)$ as an independent variable instead of the pressure $p$, we have the following Navier-Stokes problem: Find $\bar{u}, b$ and $\bar{\omega}$ in $\Omega$ such that

$$
\begin{gathered}
\nabla \cdot \bar{u}=0, \\
-\bar{u} \times \bar{\omega}+\nabla b-\frac{1}{R e} \nabla \times \bar{\omega}=\bar{f}, \\
\bar{\omega}-\nabla \times \bar{u}=0, \\
\nabla \cdot \bar{\omega}=0 .
\end{gathered}
$$

This first-order velocity-Bernoulli function-vorticity formulation is also suitable for the LSFEM.

For Cartesian co-ordinates, the system (4) is given as

$$
\begin{gathered}
\frac{\partial u}{\partial x}+\frac{\partial v}{\partial y}+\frac{\partial w}{\partial z}=0 \\
w \omega_{y}-v \omega_{z}+\frac{\partial b}{\partial x}+\frac{1}{R e}\left(\frac{\partial \omega_{z}}{\partial y}-\frac{\partial \omega_{y}}{\partial z}\right)=f_{x} \\
u \omega_{z}-w \omega_{x}+\frac{\partial b}{\partial y}+\frac{1}{R e}\left(\frac{\partial \omega_{x}}{\partial z}-\frac{\partial \omega_{z}}{\partial x}\right)=f_{y} \\
v \omega_{x}-u \omega_{y}+\frac{\partial b}{\partial z}+\frac{1}{R e}\left(\frac{\partial \omega_{y}}{\partial x}-\frac{\partial \omega_{x}}{\partial y}\right)=f_{z} \\
\omega_{x}+\frac{\partial v}{\partial z}-\frac{\partial w}{\partial y}=0 \\
\omega_{y}+\frac{\partial w}{\partial x}-\frac{\partial u}{\partial z}=0 \\
\omega_{z}+\frac{\partial u}{\partial y}-\frac{\partial v}{\partial x}=0
\end{gathered}
$$




$$
\frac{\partial \omega_{x}}{\partial x}+\frac{\partial \omega_{y}}{\partial y}+\frac{\partial \omega_{z}}{\partial z}=0
$$

For two-dimensional problems, let $\omega=\omega_{z}$, we have

$$
\begin{gathered}
\frac{\partial u}{\partial x}+\frac{\partial v}{\partial y}=0 \\
-v \omega+\frac{\partial b}{\partial x}+\frac{1}{R e} \frac{\partial \omega}{\partial y}=f_{x} \\
u \omega+\frac{\partial b}{\partial y}-\frac{1}{R e} \frac{\partial \omega}{\partial x}=f_{y} \\
\omega+\frac{\partial u}{\partial y}-\frac{\partial v}{\partial x}=0 .
\end{gathered}
$$

The velocity-Bernoulli function-vorticity formulation has some advantages from a theoretical point of view. In this formulation, the nonlinear terms $\bar{u} \times \bar{\omega}$ are of zero-order, that is, they are not related to any derivatives, and the rest of the terms constitute a linear Stokes problem. Therefore, the whole system is elliptic. This fact may simplify the nonlinear analysis of the Navier-Stokes equations.

\section{Algorithm of Solution}

The quasi-linear problem (3) (or (5), or (6)) can be linearized by the successive substitution scheme, or the Newton's scheme. For example, for the equations (3), the nonlinear convection term $u \frac{\partial u}{\partial x}$ can be linearized as $u^{0} \frac{\partial u}{\partial x}$ by using the simple substitution, or as $u^{0} \frac{\partial u}{\partial x}+u \frac{\partial u^{0}}{\partial x}-u^{0} \frac{\partial u^{0}}{\partial x}$ by using the Newton linearization scheme. Here the superscript " $0^{\text {" }}$ indicates that the value of the corresponding variable is taken from the previous calculation step. For the equations (6), the nonlinear term $-v w$ can be linearized as $-v^{0} w$ by using the simple substitution, or as $-v^{0} w-v w^{0}+v^{0} w^{0}$ by using the Newton's scheme.

The linearized first-order equations are now treated by the LSFEM[20]. The LSFEM results in symmetric and positive-definite algebraic equations. A Jacobi preconditioned conjugate gradient method is then employed to solve the linear algebraic equations. In the conjugate gradient method, the major computation is the multiplication of the global matrix with the global vector, and this can be done in an element-by-element manner without forming the global matrix[27]. In order to further save the storage, in our algorithm even the element matrices are not formed. We directly calculate the product of the element matrix and the element vector. At the same time, the Jacobi preconditioner, which consists 
of diagonal terms of the global matrix, can be easily formed. In this way we store only several global vectors, and the derivatives of the shape functions at Gaussian points for each element.

\section{Numerical Examples}

\subsection{Comparison of Two Formulations}

To compare the performance of the velocity-pressure-vorticity formulation and the velocity-Bernoulli function-vorticity formulation, we performed a series of computations for $2 \mathrm{D}$ cavity flow at different Reynolds numbers with $50 \times 50$ non-uniform bilinear elements(the mesh is the same as that used in [21]) using the following four schemes:

(a) Scheme PS, the $(u, v, p, \omega)$ formulation with simple substitution;

(b) Scheme PN, the $(u, v, p, \omega)$ formulation with Newton linearization;

(c) Scheme BS, the $(u, v, b, \omega)$ formulation with simple substitution;

(d) Scheme BN, the $(u, v, b, \omega)$ formulation with Newton linearization.

In order to eliminate any effects from iterative solvers, in all these comparison studies a direct solver was employed to solve the resulting linear algebraic system. Our numerical results reveal that, as for the speed of convergence, Scheme PS and Scheme BS are almost comparable, and Scheme PN and Scheme BN are almost comparable. However, in some cases, the schemes based on the $(u, v, b, \omega)$ formulation need a little more iterations or a closer initial guess to converge. Figure 1 shows the convergence history of the $L_{2}$-norm of residuals for the cavity flow at $R e=5000$ using different schemes. For Scheme PS and PN, the initial guess of $u$ and $v$ is taken from the results of $R e=3200$. For Scheme BN the computation started from the results of $R e=4000$. If the results of $R e=3200$ are used as the initial guess, Scheme BN does not converge. Here Scheme PS was implemented with a relaxation number of $0.8[21]$. Without relaxation Scheme PS does not converge. Scheme BS does not converge even with a relaxation number of 0.8 and the use of the results at $R e=4000$ as the initial guess. Based on the results of our numerical experiments, we are in favor of the $(u, v, p, \omega)$ formulation. In the following computation of large-scale examples, Scheme PS is used with the Jacobi preconditioned conjugate gradient method.

\subsection{D Driven Cavity Flow}

The LSFEM solution of the 2D cavity problem has already been reported in [21] by using the steady-state approach and in [24] by a time-dependent algorithm. In these previous studies, a grid of $50 \times 50$ nonuniform bilinear elements was employed. The numerical results at $R e=10000$ are in good agreement with the fine mesh $257 \times 257$ results of Ghia et al.[28].

In this work, the objectives of choosing the 2D driven cavity problem are twofold. First, we would like to show the capability of the LSFEM for large-scale problems. Second, 
we would like to find out whether improved results can be obtained by using very fine grids.

The definition of driven cavity flow is as usual. The boundary conditions for $(u, v)$ are $u=v=0$ on all solid walls and $u=1, v=0$ on the top lid. Also $p=0$ is specified at the centre of the bottom. No boundary conditions are given for the vorticity. At first we divided the cavity into $50 \times 50$ uniform bilinear elements with the size $h=0.02$. In order to take care of the singularity at the top corners, we again divided the top layer of elements into two thin layers of thickness 0.005 and 0.015 units, respectively.

At first we calculated the flow at $R e=10000$ with this $50 \times 51$ mesh. By using a kind of continuation method discussed in [21], the computation could be done on a IBM PC-386 with $4 \mathrm{M}$ bytes memory in one day. Then we refined the mesh to $100 \times 102$ elements, and interpolated the previous solution as the initial guess for the conjugate gradient method. We applied this procedure sequentially until the steady-state solution was obtained for the $400 \times 408$ mesh in which most element have the size $h=0.0025$, and in the top layer the thickness of the elements is 0.0005 . The storage required for this problem is less than $8 \mathrm{M}$ words on CRAY-YMP. The residual of each discretized equation at Gaussian points was less than $10^{-6}$.

The profile of the horizontal velocity for $R e=10000$ along the vertical center line of the driven cavity $(x=0.5)$ is illustrated in Figure 2(a). Overall, the profile compares well with that given in Ghia et al.[28], except in the boundary layers. In our results, the boundary layer phenomena are more pronounced. The computed streamlines and vorticity contours are given in Figure (2b) and (2c).

\subsection{D Driven Cavity Flow}

We choose the cubic driven cavity problem shown in Figure 3 to further test our method. This problem has been simulated by using finite difference[13,29-36] and spectral methods[37]. Due to the difficulties of existing finite element methods for large-scale computation, it may not be surprising that finite element solutions for this problem are scarce. Only Kato, Kawai and Tanahashi[38] reported the detail of numerical results for cubic cavity flows by using their GSMAC finite element methods. It should be noted that Kato et al. use the Bernoulli function and the vorticity to write the momentum equations. However, in their computation the vorticity vector is not independent, but calculated from the velocity field.

Most of numerical efforts were restrictive in mesh size and insufficient in resolution, due primarily to the limitations on numerical methods and computer resources, except Iwatsu et al. $[35,36]$, who employed very fine stretched mesh $(81 \times 81 \times 81)$.

Almost all of the previous researchers used time-marching schemes, and claimed that steady-state solutions were obtained by their own standards of convergence for $R e \leq 2000$, except Rosenfeld et al.[32] mentioned that their solution at $R e=100,1000$ was still varying 
in the lower corners. Also no Taylor-Görtler-like vortices were reported for $R e \leq 2000$ by previous researchers.

It should be mentioned that Koseff and Street[39-41] conducted systematic experiments of flow in a driven cavity. Their experiments revealed that the flow field is highly unsteady and possesses significant secondary motions (end-wall corner eddies and TaylorGörtler-like vortices). However, in all of their papers, they showed only the results for $R e=3200$, and a very important observation, that is, for what Reynolds number the flow becomes unstable, had never been reported.

In the present study, we carried out computation at $R e=100,400,1000,2000$, and 3200. $u=1, v=w=0$ were specified on the top driven surface $(y=1.0)$, and $u=v=$ $w=0$ on all solid walls(Figure 3). At the center of the bottom $(x=0.5, y=0.0, z=0.5)$, $p=0$ was specified. There were no boundary conditions on the vorticity. Three meshes were used. The first mesh consisted of $50 \times 50 \times 50$ non-uniform trilinear elements. The smallest element size had $h=0.002$ at the corners; the largest had $h=0.04$ at the center. The second mesh had $50 \times 50 \times 50$ uniform trilinear elements. The third mesh was based on the second one. In order to take care of corner singularities two layers of thin elements were added close to the top driven surface. So the third mesh had $50 \times 52 \times 50$ elements. The problems were solved using less than 13M words of memory on a CRAY-YMP.

The observations from our numerical results can be summarized as follows:

(a) Up to $R e=3200$ the flow is symmetrical about the plane $z=0.5$. This observation is consistent with the results of numerical simulation published by other researchers.

(b) The 3D cavity flow is highly complicated. For 2D driven cavity problems the small eddies appear along the boundaries. It is a common practice to use fine grids along the boundaries to capture the flow details. However, for 3D problems a pair of small vortices may be formed near the center part of the cube even for flows with moderate Reynolds numbers $(R e \geq 1000)$. Therefore, stretched $3 \mathrm{D}$ meshes may not be suitable for high-Re flows. It means that for 3D simulation we should use uniform fine grids to capture small eddies both inside and outside of the boundary layer. This makes 3D computation much more difficult than 2D computation.

(c) The $3 \mathrm{D}$ cavity flow is highly unstable. For $2 \mathrm{D}$ flows at high $R e$ number $(10000$ for the cavity flow) by using the LSFEM it is quite easy to reduce the $L_{2}$ norm of the residual(assuming the whole area of the flow domain is 1 ) to the level of $10^{-5}$. However, for the $3 \mathrm{D}$ cavity flow, we were not able to obtain the steady-state solutions for $R e \geq 1000$.

(d) The Taylor-Görtler-like vortices are observed for $R e \geq 1000$.

Some numerical results are provided in Figure 4,5 and 6. Figure 4 and 5 show the projections of velocity vectors and the contours of vorticity components on each section at $R e=.100$ and 400 . The flow patterns at $R e=100$ and 400 are in good agreement with the results of Iwatsu et al. $[35,36]$. The vorticity contours compare well with those of Hafez and 
Soliman[13]. Figure 6 clearly shows the corner vortices and TGL vortices at the bottom region of the cavity at $R e=1000$.

\section{Conclusions}

A least-squares finite element method based on the velocity-pressure-vorticity formulation is successfully extended to the solution of large-scale/3D steady-state incompressible Navier-Stokes problems. We prefer the velocity-pressure-vorticity formulation to the velocity-Bernoulli function-vorticity formulation, because the former is faster and not sensitive to initial guesses. The LSFEM generates a symmetric, positive-definite algebraic system of equations which can be robustly solved by the matrix-free conjugate gradient method. In this method there is neither upwinding, adjustable parameters, numerical boundary conditions, splitting, projection, nor artificial compressibility. Besides the finite element interpolation and the linearization, no other approximation is introduced into this method. Therefore, the solution of this method is more reliable than that of any existing method.

By using this method the steady-state solution is computed for $2 \mathrm{D}$ cavity flow at $R e=10000$ with $400 \times 408$ bilinear elements. Our results suggest that the benchmark solution of Ghia et al.[28] may not be accurate enough in the boundary layer. For 3D driven cavity flows the presence of Taylor-Görtler-like vortices is observed at $R e \geq 1000$. We hope to confirm these results with further works on the unsteady Navier-Stokes equations.

\section{Acknowledgements}

Bo-nan Jiang wishes to acknowledge the helpful discussion with prof. Gunzburger, Prof. Nicolaides and Dr. Sonnad.

\section{REFERENCES}

[1] P.S. Huyakorn, C. Taylor, R.L. Lee, and P.M. Gresho, A comparison of various mixedinterpolation finite elements in the velocity-pressure formulation of the Navier-Stokes equations, Comput.Fluids, 6, (1978) 25-35.

[2] G.F. Carey and J.T. Oden, Finite Elements: Fluid Dynamics, Vol.IV (Prentice-Hall, Englewood Cliffs, NJ, 1986).

[3] C. Cuvelier, A. Segal, A.A. Van Steenhoven, Finite Element Methods and NavierStokes Equations (Reidel, Dordrecht, 1986). 
[4] V. Girault and P.-A. Raviart, Finite Element Methods for Navier-Stokes Equations (Springer-Verlag, Berlin, 1986).

[5] M.D. Gunzburger, Finite Element Methods for Viscous Incompressible Flows (Academic Press, 1989).

[6] O. Pironneau, Finite Element Methods for Fluids (John Wiley and Sons, Chichester, 1989).

[7] R. Temam, Navier-Stokes Equations (North- Holland, Amsterdam, 1979).

[8] F. Thomasset, Implementation of Finite Element Methods for Navier-Stokes Equations (Springer, New York, 1981).

[9] P.M. Gresho, R. Sani and M. Engelman, Incompressible Flows and the Finite Element Method (Wiley, Chichester 1991).

[10] R. Glowinski and O. Pironneau, Finite element methods for Navier-Stokes equations, Annu.Rev.Fluid Mech. 24 (1992) 167-204.

[11] P.M. Gresho, R.L. Lee and R. Sani, On the time-dependent solution of the incompressible Navier-Stokes equations in two and three dimension, in C.Taylor and K.Morgan, eds., Recent Advances in Numerical Methods in Fluids, Vol.1 (Pineridge Press, 1980) p.27.

[12] T.J.R. Hughes, L.P. Franca, and G.M. Hulbert, A new finite element formulation for computational fluid mechanics: VIII. The Galerkin/least-squares method for advective-diffusive equations, Comput. Methods Appl. Mech. Engrg. 73 (1989)173189.

[13] M. Hafez and M. Soliman, Numerical solution of the incompressible Navier-Stokes equations in primitive variables on unstaggered grids, AIAA-91-1561-CP.

[14] A.J. Chorin, On the convergence of discrete approximation of the Navier-Stokes equations, Math. Comput. 22 (1968) 745-762.

[15] P.M. Gresho, On the theory of semi-implicit projection methods for viscous incompressible flow and its implementation via a finite element method that also introduces a nearly consistent mass matrix, Part 1: Theory, Inter.J.Numer.Meth.Fluids, 11 (1990) $587-620$.

[16] B. Ramaswamy, T.C. Jue and J.E. Akin, Semi-implicit and explicit finite element schemes for coupled fluid/thermal problems, Inter.J.Numer.Meth.Engng. 34 (1992) 675-696.

[17] M. Shimura and M. Kawahara, Three dimensional flow analysis using the velocity correction method, in: T.J.Chung and G.R.Karrr, eds., Finite Element Analysis in Fluids, Proceedings of the Seventh International Conference on Finite Element Methods in Flow Problems(UAH Press, Huntsville, AL, 1989) 857-862.

[18] G. Yagawa and Y. Eguchi, Finite element methods for incompressible viscous flow, JSME International Journal, 30 (1987) 1009-1017.

[19] J.T. Oden, Theory and implementation of high-order adaptive hp-methods for the analysis of incompressible viscous flows, to appear in Comput. Methods Appl. Mech. Engrg. 
[20] B.N. Jiang and L.A. Povinelli, Least-squares finite element method for fluid dynamics, Comput.Meth.Appl. Mech.Engrg. 81 (1990) 13-37.

[21] B.N. Jiang, A least-squares finite element method for incompressible Navier-Stokes problems, Inter.J.Numer.Meth.Fluids, 14 (1992) 843-859.

[22] B.N. Jiang and V. Sonnad, Least-squares solution of incompressible Navier-Stokes equations with the P-version of finite Element, NASA TM 105203, ICOMP-91-14.

[23] D. Lefebvre, J. Peraire and K. Morgan, Least squares finite element solution of compressible and incompressible flows, to be published in Int.J.Num.Methods Heat Trans.Fluid Flow.

[24] L.Q. Tang and T.T.H. Tsang, A least-squares finite element method for time-dependent incompressible flows with thermal convection, submitted to Inter.J.Numer.Meth.Fluids.

[25] B.N. Jiang and L.A. Povinelli, Optimal least-squares finite element method for elliptic problems, Comput.Meth.Appl.Mech.Engrg. in press, also available as NASA TM 105382, ICOMP-91-29.

[26] P.M. Gresho, Some current CFD issues relevant to the incompressible Navier-Stokes equations, Comput.Meth.Appl. Mech.Engrg. 87 (1991) 201-252.

[27] G.F. Carey and B.N. Jiang, Element-by-element linear and nonlinear solution schemes, Communications in Applied Numerical Methods, 2 (1986) 145-153.

[28] U. Ghia, K.N. Ghia and C.T. Shin, High-Re solutions for incompressible flow using the Navier-Stokes equation and a multigrid method, J.Comput.Phys. 48 (1982) 387-411.

[29] C.J. Freitas, R.L. Street, A.N. Findikakis and J.R. Koseff, Numerical simulation of three-dimensional flow in a cavity, Inter.J.Numer.Meth. Fluids, 5 (1985) 561-575.

[30] D.P. Hwang and H.T. Huynh, A finite difference scheme for three-dimensional steady laminar incompressible flow, Numerical Methods in Laminar and Turbulent Flow, Vol.5, Part 1, edited by Taylor, C., Habashi, W.G. and Hafez, M.M., (Pineridge Press, Swansea, UK, 1987) pp. 244-260.

[31] G. Osswald, K. Ghia, and U. Ghia, A direct algorithm for solution of three-dimensional unsteady Navier-Stokes equations, AIA A paper 87-1139.

[32] M. Rosenfeld, D. Kwak and M. Vinkour, A solution method for the unsteady and incompressible Navier-Stokes equations in generalized coordinate systems, AIA A Paper 88-0718.

[33] C.J. Freitas, and R.L. Street, Non-linear transient phenomena in a complex recirculating flow: a numerical investigation, Inter.J.Numer.Meth. Fluids, 8 (1988) 769-802.

[34] M. Hafez and M. Soliman, A velocity decomposition method for viscous incompressible flow calculations, Part II, AIAA Papers 89-1966.

[35] R. Iwatsu, K. Ishii, T. Kawamura, K. Kuwahara and J.M. Hyun, Simulation of transition to turbulence in a cubic cavity, AIAA-89-0040.

[36] R. Iwatsu, K. Ishii, T. Kawamura, K. Kuwahara and J.M. Hyun, Numerical simulation of three dimensional flow structure in a driven cavity, Fluid Dynamics Research, 5 (1989) 173-189. 
[37] H.C. Ku, R.S. Hirsh and T.D. Taylor, A pseudospectral method for solution of the three dimensional incompressible Navier-Stokes equations, J.Comput.Phys. 70 (1987) 439-462.

[38] Y. Kato, H. Kawai and T. Tanahashi, Numerical flow analysis in a cubic cavity by the GSMAC finite-element method, JSME International Journal, Series II, 33 (1990) 649-658.

[39] J.R. Koseff and R.L. Street, Visualization studies of a shear driven three-dimensional recirculation flow, J.Fluids Eng. 106 (1984) 21-29.

[40] J.R. Koseff and R.L. Street, On end wall effects in a lid-driven cavity flow, J.Fluids Eng. 106 (1984) 385-389.

[41] J.R. Koseff and R.L. Street, The lid-driven cavity flow: a synthesis of qualitative and quantitative observations, J.Fluids Eng. 106 (1984) 390-398. 


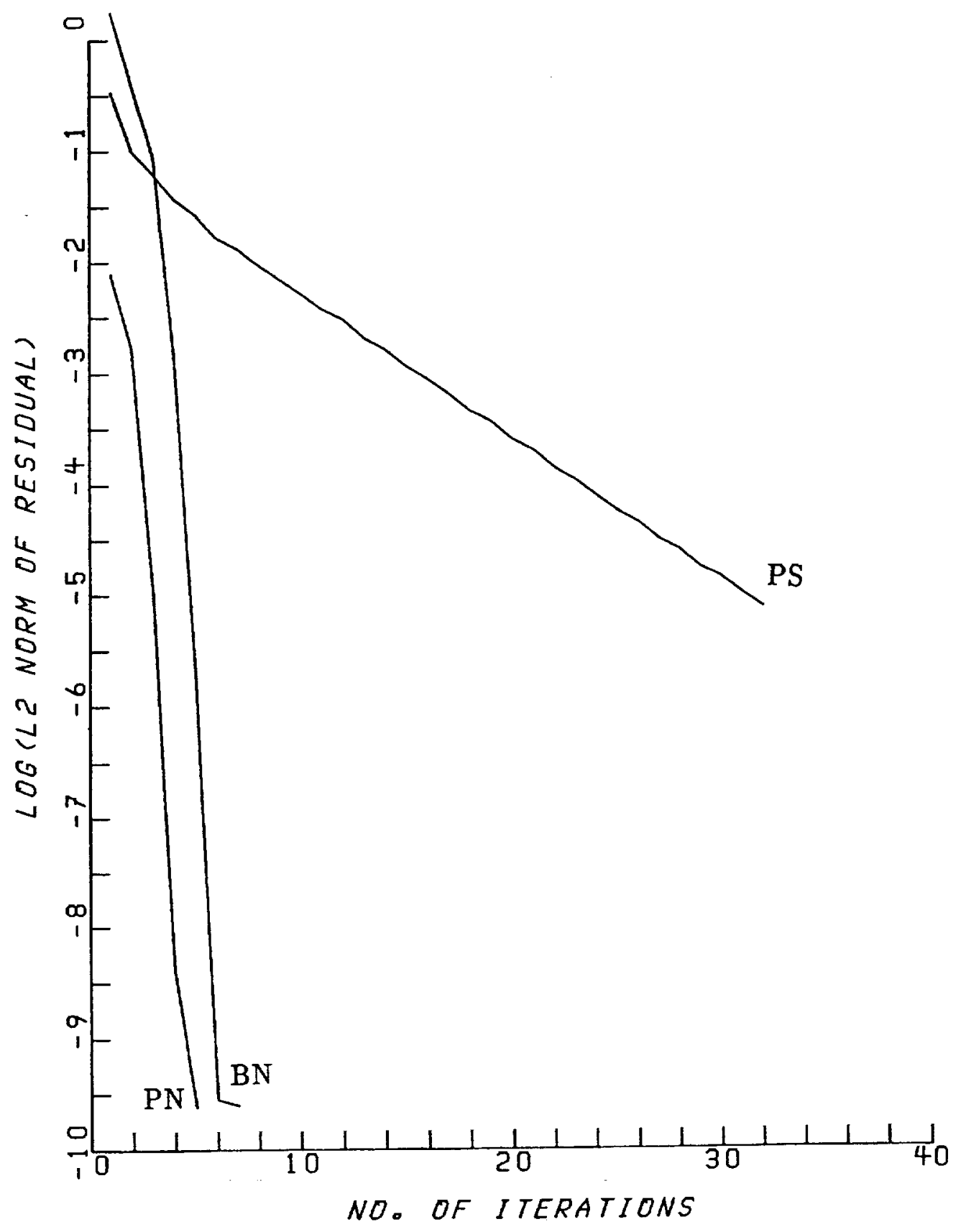

Figure 1. Convergence history for $2 \mathrm{D}$ cavity flow at $R e=5000$ 


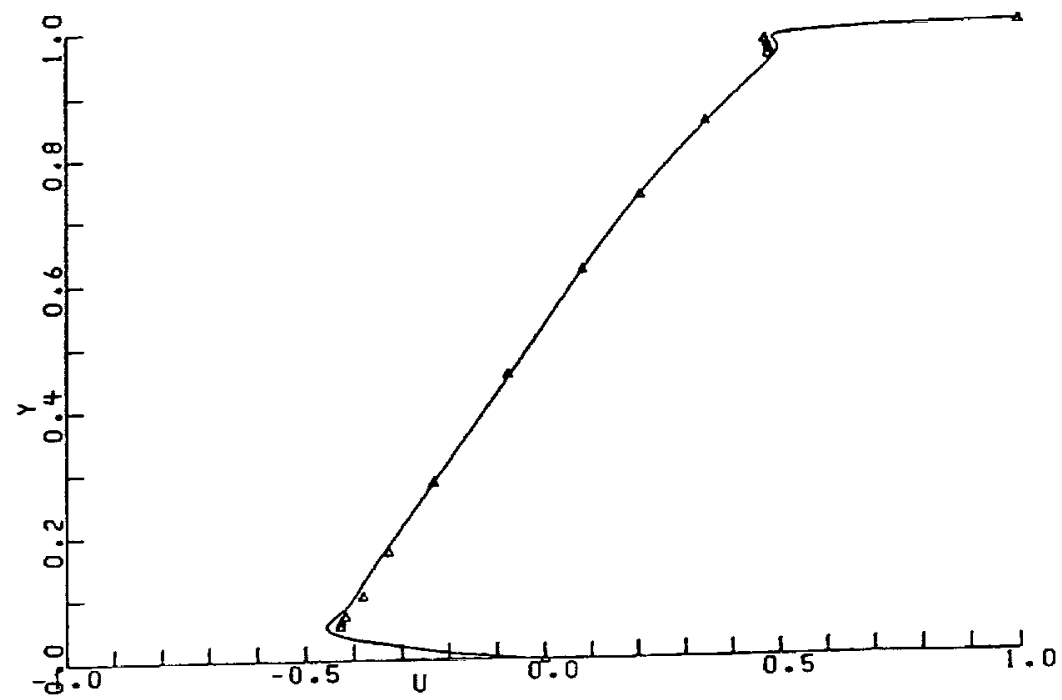

(a)

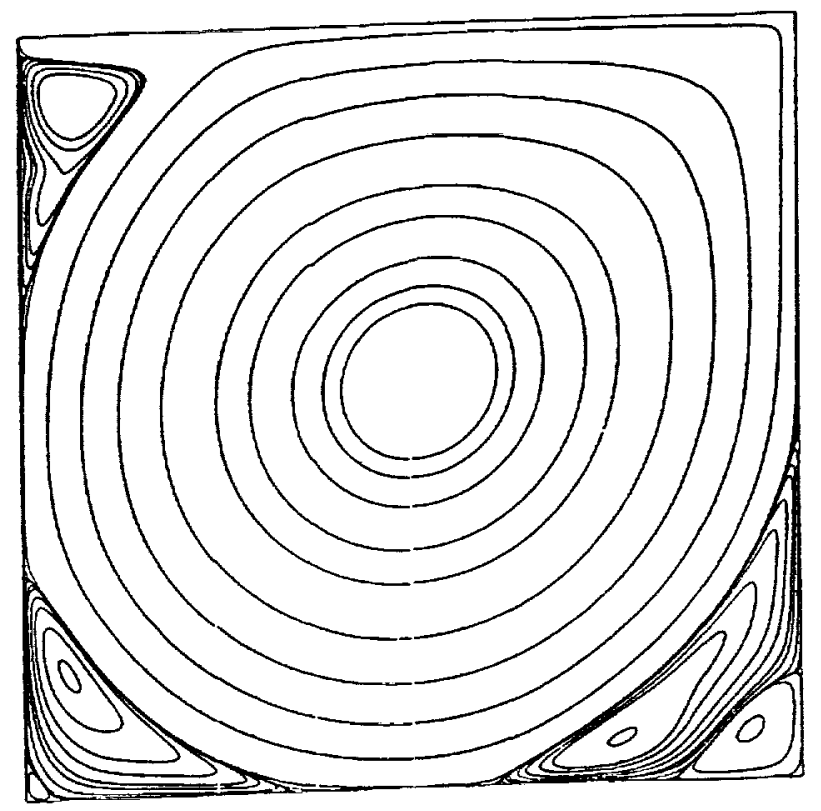

(b)

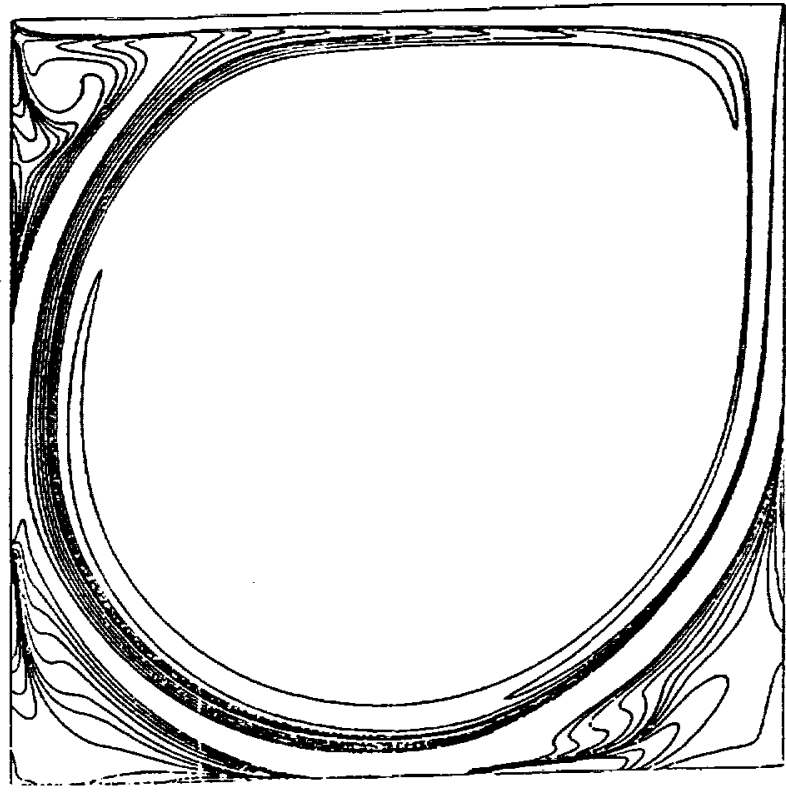

(c)

Figure 2. Numerical results for $2 \mathrm{D}$ cavity flow at $R e=10000$

(a) Horizontal velocity profile, present $(401 \times 409), \triangle \mathrm{Ghia}[28](257 \times 257)$ (b) Streamlines, (c) Vorticity contours. 


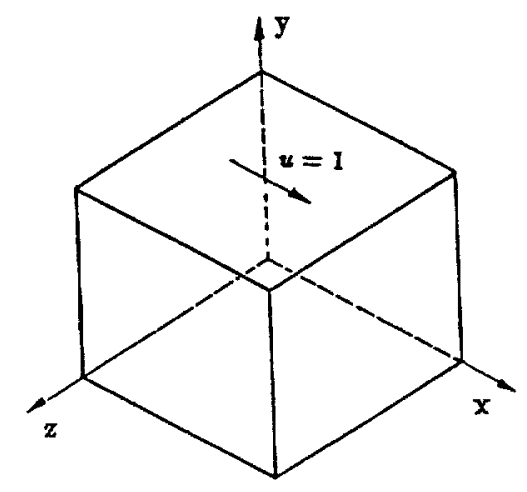

Figure 3. Geometry and boundary conditions of cubic cavity flow 


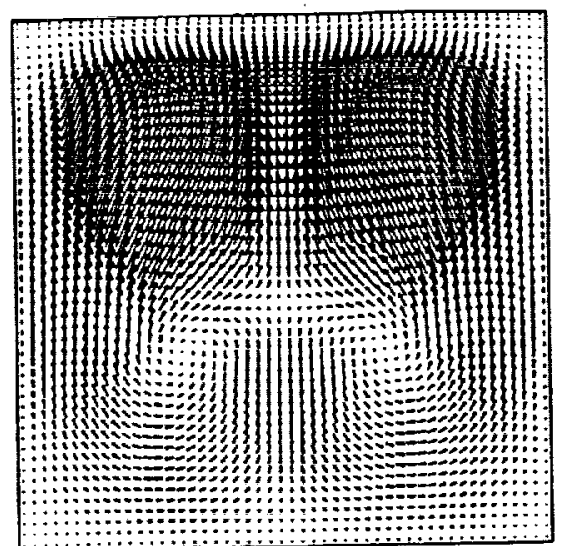

(a) Velocity vector at $x=0.5$

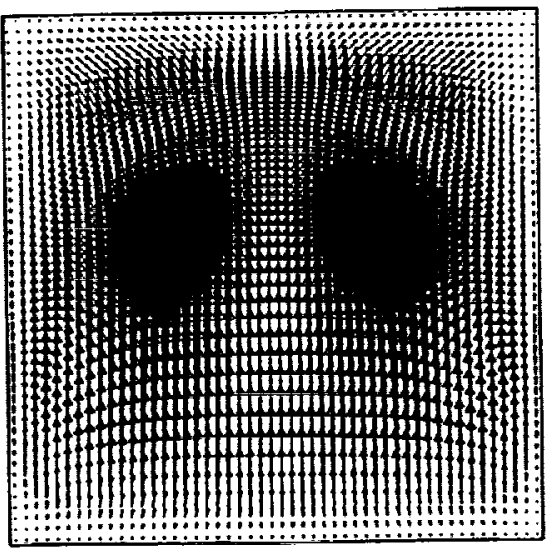

(d) Velocity vector at $y=0.5$

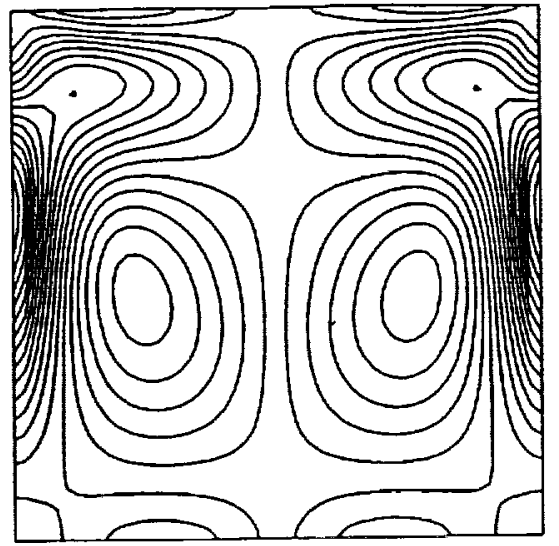

(b) Vorticity $\left(\omega_{x}\right)$ contours at $x=0.5$

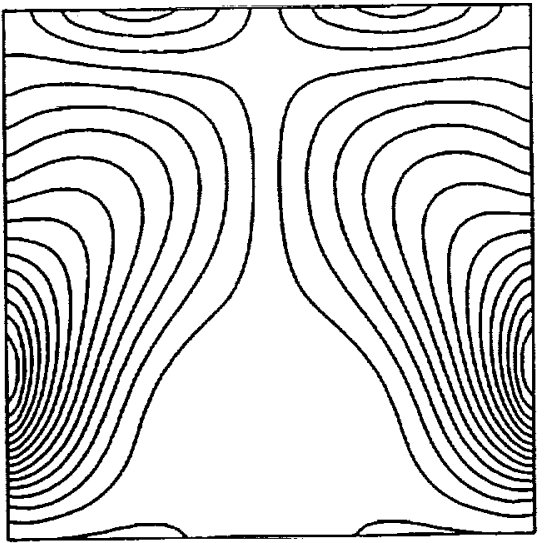

(e) Vorticity $\left(\omega_{y}\right)$ contours at $y=0.5$

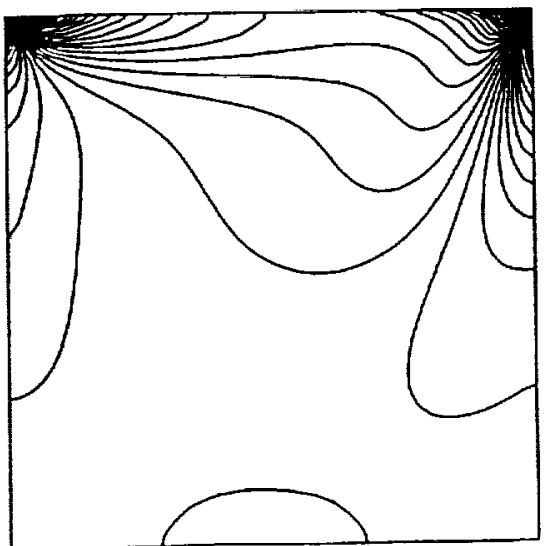

(h) Vorticity $\left(\omega_{z}\right)$ contours at $z=0.5$

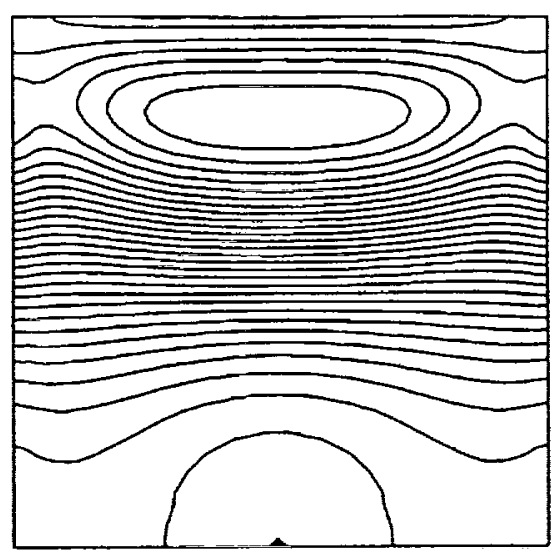

(c) Pressure contours at $x=0.5$

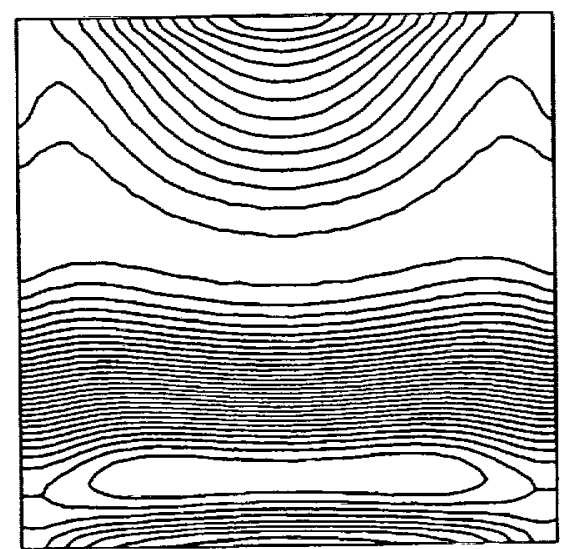

(f) Pressure contours at $y=0.5$

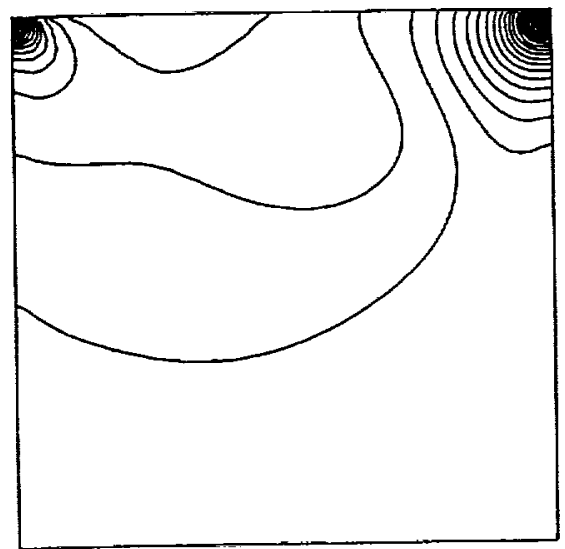

(i) Pressure contours at $z=0.5$

(g) Velocity vector at $z=0.5$

Figure 4. Numerical results for $3 \mathrm{D}$ cavity flow at $R e=100$ 


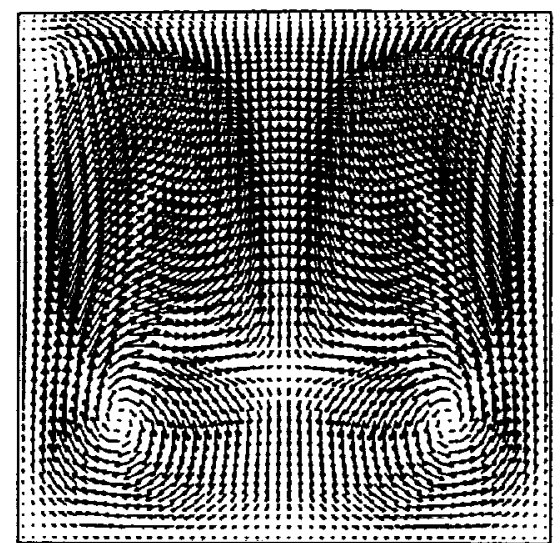

(a) Velocity vector at $x=0.5$

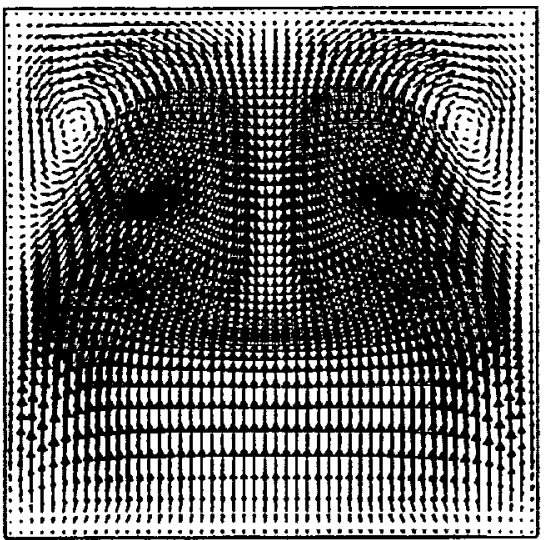

(d) Velocity vector at $y=0.5$

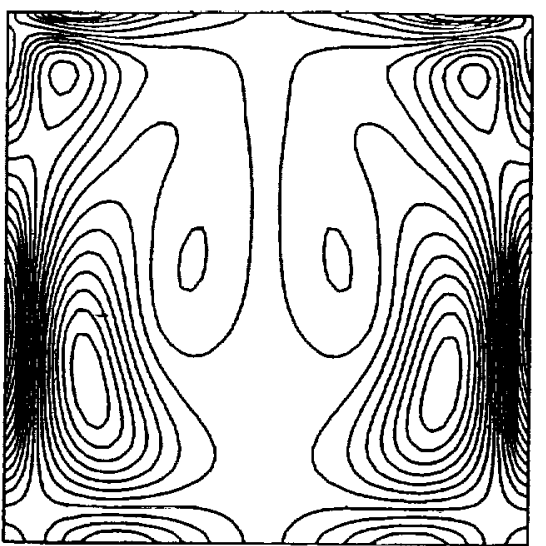

(b) Vorticity $\left(\omega_{x}\right)$ contours at $x=0.5$

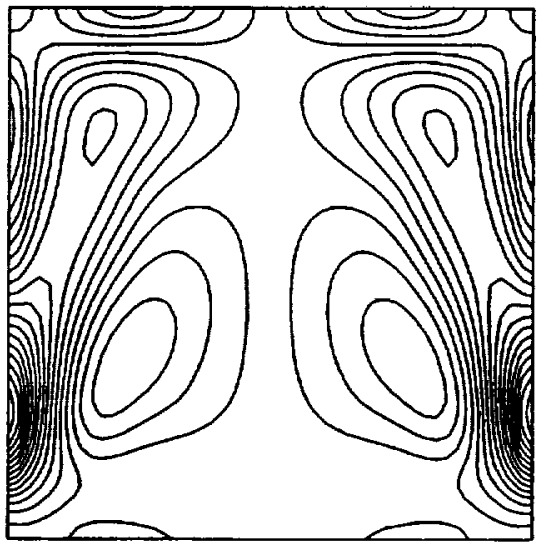

(e) Vorticity $\left(\omega_{y}\right)$ contours at $y=0.5$

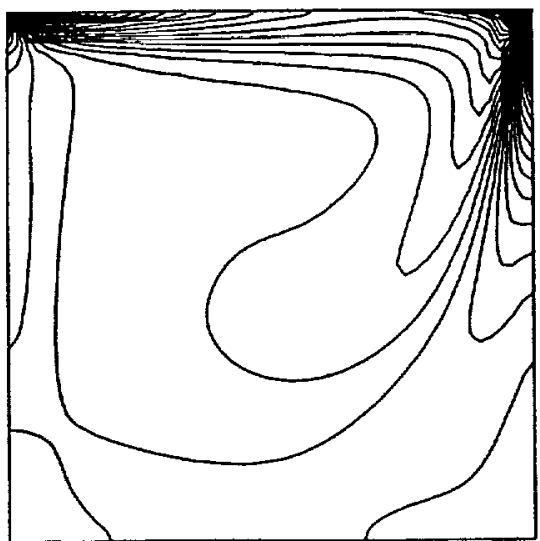

(h) Vorticity $\left(\omega_{z}\right)$ contours at $\mathrm{z}=0.5$

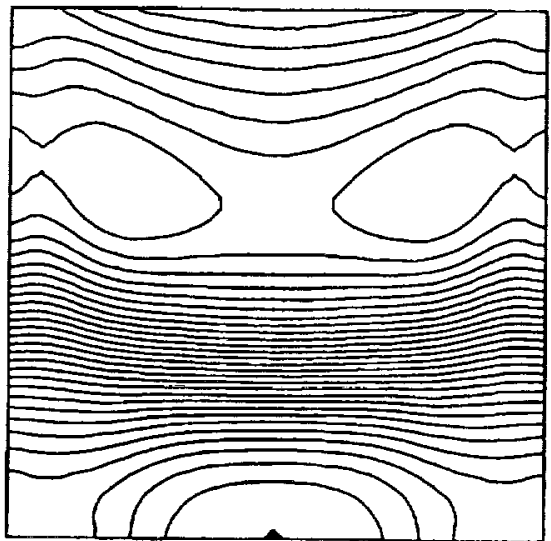

(c) Pressure contours at $x=0.5$

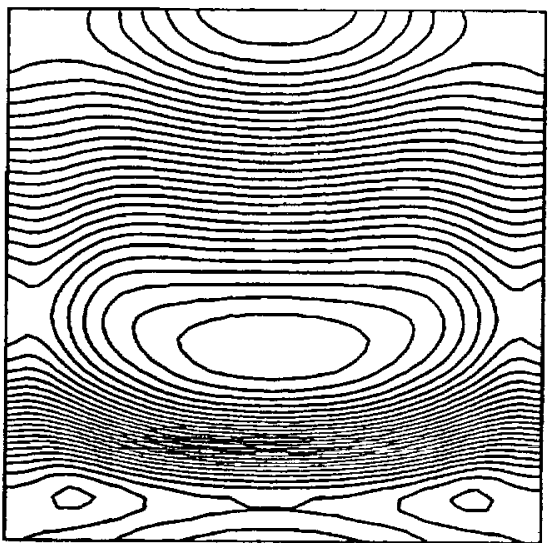

(f) Pressure contours at $y=0.5$

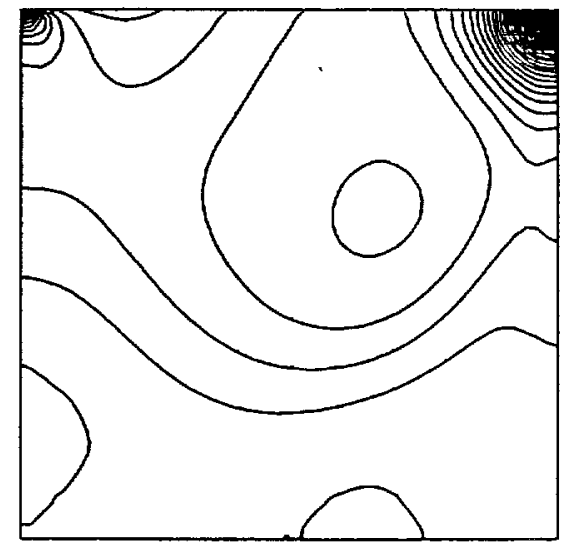

(i) Pressure contours at $z=0.5$

(g) Velocity vector at $\mathrm{z}=0.5$

Figure 5. Numerical results for 3D c8vity flow at $R e=400$ 


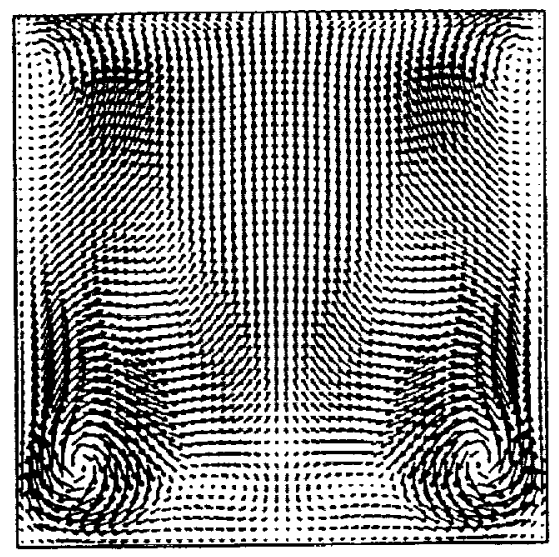

(a) Velocity vector at $x=0.5$

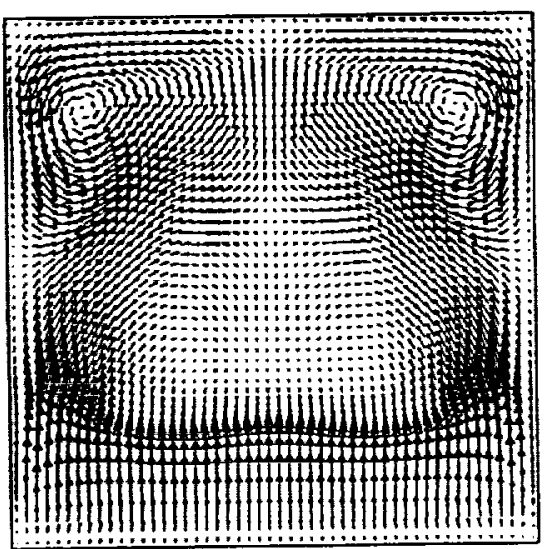

(d) Velocity vector at $y=0.5$

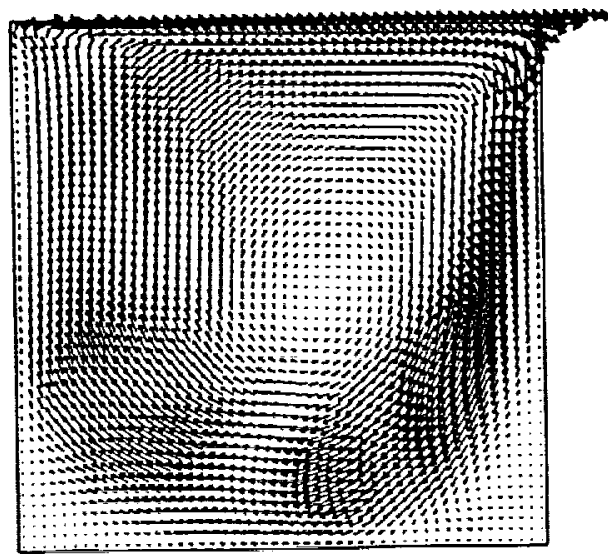

(g) Velocity vector at $\mathrm{z}=0.5$

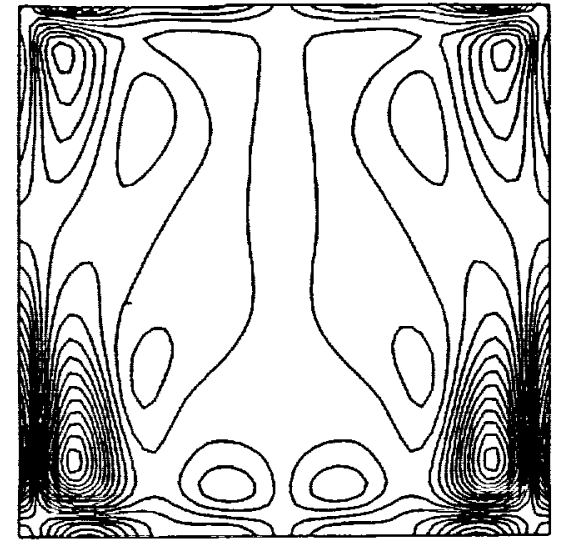

(b) Vorticity $\left(\omega_{x}\right)$ contours at $x=0.5$

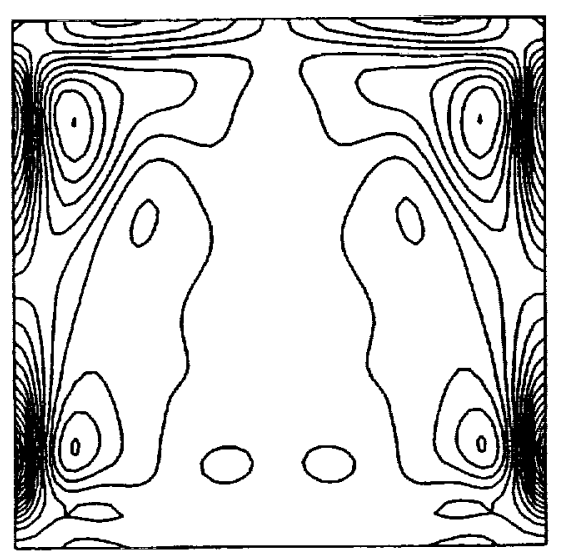

(e) Vorticity $\left(\omega_{y}\right)$ contours at $y=0.5$

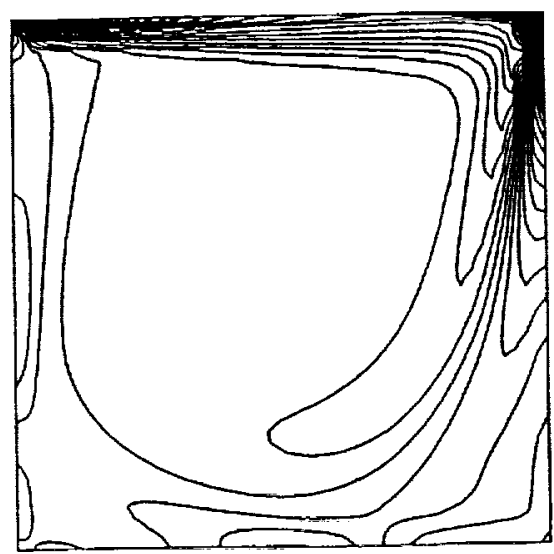

(h) $\operatorname{Vorticity}\left(\omega_{z}\right)$ contours at $z=0.5$

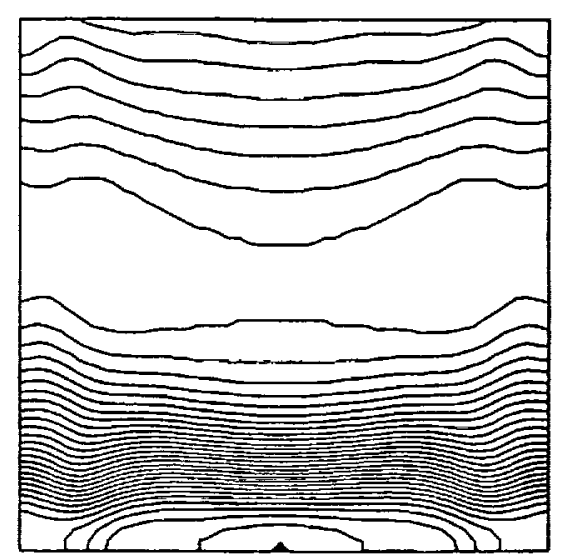

(c) Pressure contours at $x=0.5$

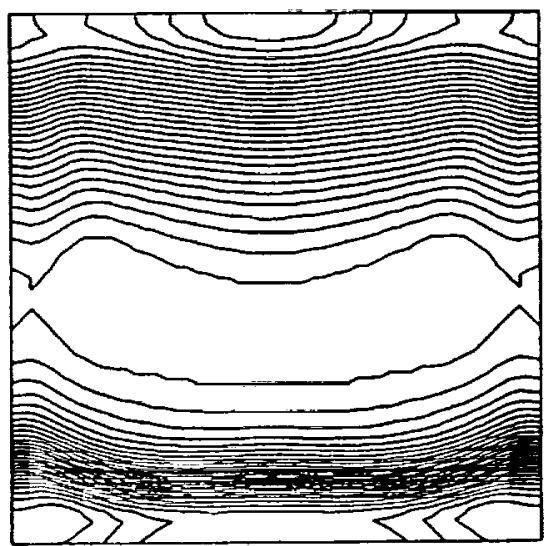

(f) Pressure contours at $y=0.5$

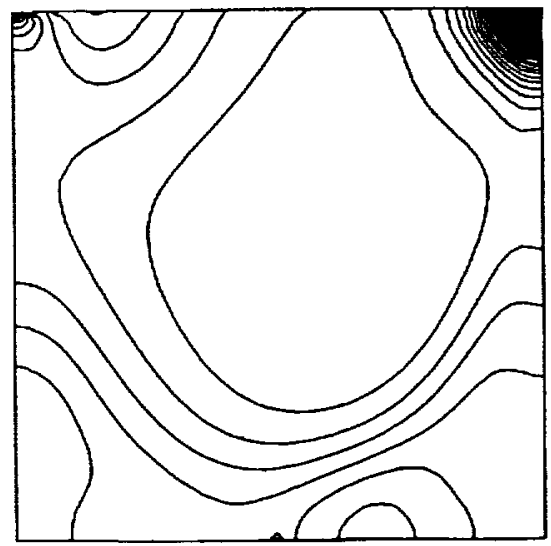

(i) Pressure contours at $\mathrm{z}=0.5$

Figure 6. Numerical results for $3 \mathrm{D}$ cavity flow at $R e=1000$ 

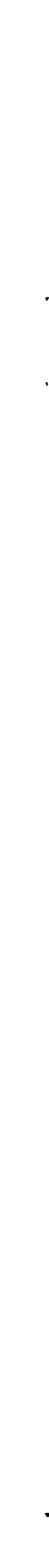
Public reporting burden for this collection of information is estimated to average 1 hour per response, including the time for reviewing instructions, searching existing data sources, gathering and maintaining the data needed, and completing and reviewing the collection of information. Send comments regarding this burden estimate or any other aspect of this collection of information, including suggestions for reducing this burden, to Washington Headquarters Sences, Directorate for intomation Operations and Report, Washington. DC 20503. Davis Highay, Sutre 1204, Artington, VA 22202-4302, and to the Office of Management and Budget, Paperwork Ri

\begin{tabular}{|l|c|c|}
\hline 1. AGENCY USE ONLY (Leave blank) & $\begin{array}{c}\text { 2. REPORT DATE } \\
\text { March } 1993\end{array}$ & $\begin{array}{r}\text { 3. REPORT TYPE AND DATES COVERED } \\
\text { Technical Memorandum }\end{array}$
\end{tabular}

4. TITLE AND SUBTITLE

5. FUNDING NUMBERS

Large-Scale Computation of Incompressible Viscous Flow by Least-Squares

Finite Element Method

6. AUTHOR(S)

Bo-nan Jiang, T.L. Lin, and Louis A. Povinelli

WU-505-62-21

7. PERFoRMING ORGANRATION NAME(S) AND ADDAESS(ES)

8. PERFORIING ORGANIZATION REPORT NUMBER

National Aeronautics and Space Administration

Lewis Research Center

E-7667

Cleveland, Ohio 44135-3191

9. SPONSORING/MONITORING AGENCY NAMES(S) AND ADDRESS(ES)

10. SPONSORINGMONTORING AGENCY REPORT NUMBER

National Aeronautics and Space Administration

Washington, D.C. 20546-0001

NASA TM-105904

ICOMP-93-06

\section{SUPPLEMENTARY NOTES}

Bo-nan Jiang, Institute for Computational Mechanics in Propulsion, NASA Lewis Research Center, (work funded under NASA

Cooperative Agreement NCC3-233). T.L. Lin, Livermore Software Technology Corporation, Livermore, California 94550.

Louis A. Povinelli, NASA Lewis Research Center. ICOMP Program Director, Louis A. Povinelli, (216) 433-5818.

12a. DISTRIBUTIONIAVAILABILITY STATEMENT

12b. DISTRIBUTION CODE

Unclassified - Unlimited

Subject Category 64

\section{ABSTRACT (Maximum 200 words)}

The least-squares finite element method (LSFEM) based on the velocity-pressure-vorticity formulation is applied to large-scale/three-dimensional steady incompressible Navier-Stokes problems. This method can accommodate equalorder interpolations, and results in symmetric, positive definite algebraic system which can be solved effectively by simple iterative methods. The first-order velocity-Bernoulli function-vorticity formulation for incompressible viscous flows is also tested. For three-dimensional cases, an additional compatibility equation, i.e., the divergence of vorticity vector should be zero, is included to make the first-order system elliptic. The simple substitution or the Newton's method is employed to linearize the partial differential equations, the LSFEM is used to obtain discretized equations, and the system of algebraic equations is solved using the Jacobi preconditioned conjugate gradient method which avoids formation of either element or global matrices (matrix-free) to achieve high efficiency. To show the validity of this scheme for large-scale computation, we give numerical results for $2 \mathrm{D}$ driven cavity problem at $\mathrm{Re}=10000$ with $408 \times 400$ bilinear elements. The flow in a $3 \mathrm{D}$ cavity is calculated at $\operatorname{Re}=100,400$, and 1,000 with $50 \times 50 \times 50$ trilinear elements. The Taylor-Görtler-like vortices are observed for $\operatorname{Re}=1,000$.

\section{SUBJECT TERMS}

Least squares; Finite element; Incompressible; Navier-stokes; Viscous flow; Velocitypressure-vorticity; Three dimensional; Driven cavity

15. NUMBER OF PAGES

17. SECUATY CLASSIFICATION OF REPORT Unclassified

18. SECURITY CLASSIFICATION
OF THIS PAGE
Unclassified
19. SECUAITY CLASSIFICATHON OF ABSTRACT Unclassified
20. LIMITATION OF ABSTRACT 